\title{
Mortality in the Protected Leopard's Population, Uttarakhand, North India: A Free-Ranging Wildlife Species in Threat
}

\author{
Ritesh Joshi $^{1, *}$, Rajendra Agarwal ${ }^{2}$ \\ ${ }^{1}$ Conservation and Survey Division, Ministry of Environment and Forests, Govt. of India, Paryavaran Bhawan, CGO Complex, New \\ Delhi-110 003, India \\ ${ }^{2}$ Wildlife Protection Society of India, Uttarakhand Region, Kankhal, Haridwar, 249 401, Uttarakhand, India
}

\begin{abstract}
Large cats are vulnerable to local extinction in fragmented landscapes mainly due to large scope developmental and anthropogenic activities. Present study highlights the mortality of protected leopard's population in Uttarakhand state, north-west India. In between January 2009 to October 2010, 78 leopards have died due to various reasons accounted primarily for unnatural deaths. Maximum deaths occurred in between February 2009 \& April 2010 and notably 37 leopards died since January, 2010. The mortality rate for females was significantly higher than for males. Notably, 11 leopards were found dead scrambled in trap and some deaths occurred while providing treatment after rescuing the animal. In addition, 21 cases of leopard's poaching (illegal wildlife trade) were also documented in between January 2009 to March 2010 in which 35 leopard's skins were recovered, which highlighted that poaching is also ongoing in some remote areas. Status of man-animal conflict in Uttarakhand is severely increasing; in between November 2000 to December 2008, 180 people died in leopard's attack, whereas 343 were injured during encounters and leopard's attacks. On the other hand, 394 leopards died due to other reasons during the said period and 50 were declared as man-eater, which were shot dead or translocated to other protected habitat. Here, we report on the mortality in protected leopard population of Uttarakhand state and conservation status. Such reports are highly required to know the status and our competence in illustrating success and failures of wildlife rescue operations besides in conservation of an endangered wildlife.
\end{abstract}

Keywords Conservation, Leopard, Mortality, Protected Habitat, Threat, Uttarakhand

\section{Introduction}

Leopard (Panthera pardus) is widely distributed wild big cat and listed as an endangered species in IUCN Red List of Threatened Mammals and categorized under Schedule 1 of "Wildlife Protection Act 1972". In between January 2009 to October 2010, this species has dwindled drastically in Uttarakhand state and its existing populations are at high risk (Figure 1). One of the main reasons for this decline is the degradation of the habitat and loss of connectivity among different protected habitats. In Uttarakhand, Rajaji-Corbett wildlife corridor is the strong hold of leopards with a population of 608 (census 2005, Figure 5 A\&B), which also includes the population in Rajaji National Park, Corbett Tiger Reserve (including Sonanadi Wildlife Sanctuary and Kalagarh Tiger Reserve), Lansdowne, Dehradun, Haridwar, Ramnagar, Terai East, Terai West, Terai Central and

* Corresponding author:

ritesh_joshi2325@yahoo.com (Ritesh Joshi)

Published online at http://journal.sapub.org/ije

Copyright (C) 2012 Scientific \& Academic Publishing. All Rights Reserved
Haldwani forests divisions. Remarkably, in between January 2009 and April 11, 2010, 78 leopards have died at different locations of Uttarakhand state and most of the deaths occurred in protected habitats (Table 1).

\section{Causes of Mortality}

The specific reason of maximum number of leopard's deaths was not clearly known because of old carcass found. This has accounted for 30 leopards (38.4\%), which were found dead at different locations of the state (Figure $5 \mathrm{C} \& \mathrm{D}$ ). Ground data revealed that $24.3 \%$ (19) leopards died due to poaching / captured in trap (in some cases died during care after rescued from trap, Figure 5 E) whereas 20.5\% (16) leopards were declared as man-eater and had been shot dead. Road accidental deaths accounted for $6.4 \%$ (05), killed by villager in self-defense accounted for $3.9 \%(03)$ and train accidental deaths accounted for $1.3 \%(01)$. A total of $3.9 \%$ (03) animal had been died in internal fight whereas $1.3 \%(01)$ killed in wildfires (Table 1, Figure 2, $5 \mathrm{~F} \& \mathrm{G}$ ). However, it was said in some cases that leopard died because of disease/ illness, but we assumed all these cases under unknown 
category. It is quite difficult to find out accurate reason of death especially when any old carcass is found, even after the

sons. However, exact death reason could be known tentapost-mortem; it is very difficult to trace accurate death rea-

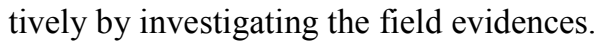

Table 1. Leopard's mortality in Uttarakhand since January 2009

\begin{tabular}{|c|c|c|c|c|}
\hline 1. & $\begin{array}{l}\text { January 01, } \\
2009 \\
\end{array}$ & Terai West Forest Division & - & Found injured, died during care \\
\hline 2. & $\begin{array}{c}\text { January } 22 \\
2009 \\
\end{array}$ & $\begin{array}{l}\text { Pokhaal, Kotdwar, Lansdowne Forest } \\
\text { Division }\end{array}$ & $\begin{array}{l}\text { Female, } \\
03 \text { years }\end{array}$ & Shot-dead, declared as man-eater \\
\hline 3. & $\begin{array}{l}\text { February } \\
03,2009\end{array}$ & $\begin{array}{c}\text { Rajawala, Selaqui, Dehradun Forest } \\
\text { Division }\end{array}$ & - & Found dead \\
\hline 4. & $\begin{array}{l}\text { February } \\
06,2009\end{array}$ & Almora Forest Division & - & Internal Fight \\
\hline 5. & $\begin{array}{l}\text { February } \\
08,2009\end{array}$ & Almora Forest Division & Male, & Found dead, Unknown \\
\hline 6. & $\begin{array}{l}\text { February } \\
13,2009\end{array}$ & $\begin{array}{l}\text { Gopeshwar-Chamoli Route, Nanda } \\
\text { Devi Biosphere Reserve }\end{array}$ & - & Trapped in clutch wire, rescued, died during care \\
\hline 7. & $\begin{array}{l}\text { February } \\
16,2009 \\
\end{array}$ & Almora Forest Division &,$- \mathrm{Cub}$ & Found Dead, Unknown \\
\hline 8. & $\begin{array}{l}\text { February } \\
16,2009\end{array}$ & Nainital Forest Division &,$- \mathrm{Cub}$ & Found Dead, Unknown \\
\hline 9. & $\begin{array}{l}\text { February } \\
17,2009 \\
\end{array}$ & $\begin{array}{l}\text { Ghamandpur, Ranipokhri, Barkot } \\
\text { Range, Dehradun, Forest Division }\end{array}$ & Male, - & Found dead \\
\hline 10. & $\begin{array}{l}\text { February } \\
18,2009 \\
\end{array}$ & $\begin{array}{l}\text { Paathisain area, Dhumakot, Pauri } \\
\text { Forest Division } \\
\end{array}$ & Male, - & Found dead \\
\hline 11. & $\begin{array}{l}\text { February } \\
18,2009 \\
\end{array}$ & $\begin{array}{l}\text { Paathisain area, Dhumakot, Pauri } \\
\text { Forest Division }\end{array}$ & Female, - & Found dead \\
\hline 12. & $\begin{array}{l}\text { February } \\
19,2009\end{array}$ & Pokhaal, Pauri Forest & - & Shot-dead by shooter; declared as man-eater \\
\hline 13. & $\begin{array}{l}\text { February } \\
19,2009\end{array}$ & Gairsain, Chamoli Forest & - & Shot-dead by shooter; declared as man-eater \\
\hline 14. & $\begin{array}{l}\text { February } \\
19,2009\end{array}$ & $\begin{array}{c}\text { Khatima-Banbasa Route, Banbasa } \\
\text { Forest }\end{array}$ & Female, - & Road accident \\
\hline 15. & $\begin{array}{l}\text { March 05, } \\
2009\end{array}$ & Kholiagaon, Bageshwar Forest & - & Shot-dead, declared as man-eater \\
\hline 16. & $\begin{array}{l}\text { April 12, } \\
2009 \\
\end{array}$ & Gairsain, Chamoli Forest & Female, - & Shot-dead, declared as man-eater \\
\hline 17. & $\begin{array}{l}\text { April 26, } \\
2009\end{array}$ & $\begin{array}{l}\text { Gadgadia Range, Haldwani Forest } \\
\text { Division }\end{array}$ & - & Found dead \\
\hline 18. & $\begin{array}{l}\text { May } 13, \\
2009\end{array}$ & $\begin{array}{c}\text { Chopra, Raipur Range, Mussoorie } \\
\text { Forest Division } \\
\end{array}$ & Female. - & Shot-dead by shooter, declared as man-eater \\
\hline 19. & $\begin{array}{c}\text { May } 20 \\
2009\end{array}$ & $\begin{array}{l}\text { Haridwar-Roorkee route, near to } \\
\text { Pathri rau, Haridwar Forest Division }\end{array}$ & - & Road accident \\
\hline 20. & $\begin{array}{l}\text { June } 09 \\
2009 \\
\end{array}$ & Tol village, Devprayag & $\begin{array}{l}\text { Female, } \\
08 \text { years }\end{array}$ & Shot dead, declared as man-eater \\
\hline 21. & $\begin{array}{l}\text { June } 28 \\
2009\end{array}$ & Devprayag & - & Shot dead, declared as man-eater \\
\hline 22. & $\begin{array}{l}\text { June } \ldots \text {, } \\
2009\end{array}$ & $\begin{array}{l}\text { Sikroda, Khanpur Range, Haridwar } \\
\text { Forest Division }\end{array}$ & - & Found dead \\
\hline 23. & $\begin{array}{l}\text { July } 01 \\
2009\end{array}$ & Premnagar, Dehradun Forest Division & Female, - & Shot dead, declared as man-eater \\
\hline 24. & $\begin{array}{l}\text { July } 14 \\
2009\end{array}$ & Bharpur patti, Devprayag, Tehri & - & Killed by shooter (declared as man-eater) \\
\hline 25. & $\begin{array}{l}\text { July } 15 \\
2009\end{array}$ & $\begin{array}{l}\text { Kudkawala, Haridwar Forest Divi- } \\
\text { sion }\end{array}$ & $\begin{array}{l}\text { Male, } 05 \\
\text { years }\end{array}$ & Found dead, Poaching (bullets were found over to body) \\
\hline 26. & $\begin{array}{l}\text { July } 16 \\
2009\end{array}$ & $\begin{array}{c}\text { Kumrada village, Chinyalisaur, } \\
\text { Uttarkashi }\end{array}$ & $\begin{array}{l}\text { Female, } \\
04 \text { years }\end{array}$ & Killed by villager during attack/self-defence \\
\hline
\end{tabular}


Ritesh Joshi et al:: Mortality in the Protected Leopard's Population, Uttarakhand,

North India: A Free-Ranging Wildlife Species in Threat

\begin{tabular}{|c|c|c|c|c|}
\hline 27. & $\begin{array}{l}\text { July } 17 \\
2009\end{array}$ & Khandah, Pauri & - & Brutally killed by villagers \\
\hline 28. & $\begin{array}{c}\text { July } 23 \text {, } \\
2009\end{array}$ & Nainital Zoo & $\begin{array}{l}\text { Female, } \\
04 \text { years }\end{array}$ & Shifted from Birla Forest, rescued, finally died \\
\hline 29. & $\begin{array}{l}\text { July } 27 \\
2009\end{array}$ & Gairsain area, Chamoli Forest & - & Shot dead, declared as man-eater \\
\hline 30. & $\begin{array}{l}\text { August } 16 \\
2009\end{array}$ & East Jhandi chaur, Kotdwar Forest & - & Found injured, died during care \\
\hline 31. & $\begin{array}{l}\text { August } 19 \\
2009\end{array}$ & Chiniyalisaur, Uttarkashi & - & Found injured, died during rescue \\
\hline 32. & $\begin{array}{l}\text { August 21, } \\
2009\end{array}$ & Gopeshwar, Chamoli Forest Division & - & Found injured, died during rescue operation \\
\hline 33. & $\begin{array}{l}\text { August 21, } \\
2009\end{array}$ & Gairsain, Chamoli Forest & $\begin{array}{c}\text { Male, } 05 \\
\text { years }\end{array}$ & Shot dead, declared as man-eater \\
\hline 34. & $\begin{array}{l}\text { October 07, } \\
2009\end{array}$ & $\begin{array}{l}\text { Motasaal are, Sonanadi Wildlife } \\
\text { Sanctuary Kalagarh Tiger Reserve }\end{array}$ & Female, - & Found dead \\
\hline 35. & $\begin{array}{l}\text { October 13, } \\
2009\end{array}$ & $\begin{array}{c}\text { Chaukhutia Block, Almora Forest } \\
\text { Division }\end{array}$ & - & Shot dead, declared as man-eater \\
\hline 36. & $\begin{array}{l}\text { October } 15 \\
2009\end{array}$ & $\begin{array}{c}\text { Yamkeshwar Block, Pauri Forest } \\
\text { Division }\end{array}$ & Male,- & Shot dead, declared as man-eater \\
\hline 37. & $\begin{array}{l}\text { October } 23 \\
2009\end{array}$ & $\begin{array}{l}\text { Kaladhungi Range, Ramnager Forest } \\
\text { Division }\end{array}$ & - & Found dead, internal fight \\
\hline 38. & $\begin{array}{c}\text { November } \\
07,2009\end{array}$ & $\begin{array}{l}\text { Bhardaar are, Jakholi Bl., Rudra- } \\
\text { prayag Forest Division }\end{array}$ & Female, - & Shot dead, declared as man-eater \\
\hline 39. & $\begin{array}{c}\text { November } \\
12,2009\end{array}$ & Kansrao, Rajaji National Park & Female, - & Train accident \\
\hline 40. & $\begin{array}{c}\text { November } \\
20,2009\end{array}$ & $\begin{array}{l}\text { Chini Forest, Khatima Range, Terai } \\
\text { East, Forest Division }\end{array}$ & Male, - & $\begin{array}{l}\text { Found dead, carcass found in a nallah; Haemorrhage of } \\
\text { intestine/bleeding? }\end{array}$ \\
\hline 41. & $\begin{array}{c}\text { December } \\
24,2009\end{array}$ & $\begin{array}{c}\text { Chauharpura Range, Barwa village, } \\
\text { Dehradun Forest Division } \\
\end{array}$ & - & Found dead; 03 nails missing \\
\hline 42. & $\begin{array}{l}\text { January } 06 \\
2010\end{array}$ & $\begin{array}{l}\text { Chaukuni Forest, Champawat Forest } \\
\text { Division }\end{array}$ & $\begin{array}{l}\text { Female, } \\
02 \text { years }\end{array}$ & Found dead, Unknown \\
\hline 43. & $\begin{array}{c}\text { January } 07, \\
2010 \\
\end{array}$ & $\begin{array}{c}\text { Rishikesh Forest, Dehradun Forest } \\
\text { Division }\end{array}$ & $\begin{array}{l}\text { Female, } \\
02 \text { years }\end{array}$ & Road accident \\
\hline 44. & $\begin{array}{c}\text { January } 12, \\
2010\end{array}$ & $\begin{array}{c}\text { Patrampur, south Jaspur, Terai West } \\
\text { Forest Division } \\
\end{array}$ & $\begin{array}{l}\text { Female, } \\
04 \text { years }\end{array}$ & Found Dead, Unknown \\
\hline 45. & $\begin{array}{l}\text { January } 15 \\
2010\end{array}$ & $\begin{array}{c}\text { Forest near to Buggawala village, } \\
\text { Khanpur Forest, Haridwar Forest } \\
\text { Division } \\
\end{array}$ & - & Found Dead, Unknown (post-mortem report - Disease) \\
\hline 46. & $\begin{array}{l}\text { January } 21, \\
2010 \\
\end{array}$ & $\begin{array}{l}\text { Haldwani Forest, Terai Central Forest } \\
\text { Division } \\
\end{array}$ &,- 03 years & Road accident \\
\hline 47. & $\begin{array}{c}\text { January } 22, \\
2010 \\
\end{array}$ & $\begin{array}{l}\text { Jhajra Forest, Dehradun Forest Divi- } \\
\text { sion }\end{array}$ & - & Shooted down [declared as man-eater] \\
\hline 48. & $\begin{array}{c}\text { January } 24, \\
2010 \\
\end{array}$ & $\begin{array}{l}\text { Forest near to Bosaan village, Chak- } \\
\text { rata Forest Division }\end{array}$ & - & Found dead, unknown \\
\hline 49. & $\begin{array}{l}\text { January } 27, \\
2010\end{array}$ & $\begin{array}{l}\text { Kota Forest, Ramnagar Forest Divi- } \\
\text { sion (Paatkot village) }\end{array}$ & $\begin{array}{l}\text { Male, } 06 \\
\text { years }\end{array}$ & Found dead, Internal fight \\
\hline 50. & $\begin{array}{l}\text { January } 29, \\
2010\end{array}$ & Bakhli village, Chaukhutia, Almora &,- 01 year & Found dead, unknown \\
\hline 51. & $\begin{array}{l}\text { January } 29, \\
2010\end{array}$ & Malsi Deer Park, Dehradun &,- 01 year & Disease, Brutally rescued from Survey of India campus \\
\hline 52. & $\begin{array}{l}\text { February } \\
07,2010\end{array}$ & $\begin{array}{l}\text { Gaulapaar area, near to Kalichaur } \\
\text { river, Chakata Range, Ratighat } \\
\text { Compt., Haldwani Forest Division }\end{array}$ & $\begin{array}{l}\text { Male, } 05 \\
\text { years }\end{array}$ & Found dead \\
\hline 53. & $\begin{array}{l}\text { February } \\
07,2010\end{array}$ & $\begin{array}{l}\text { Hempur forest, Aampokhra Range, } \\
\text { Terai west Forest Division }\end{array}$ & - & $\begin{array}{l}\text { Captured in fencing, rescued during night of } 06^{\text {th }} \text { but finally } \\
\text { died; fencing of agriculture farm of Remount training school } \\
\& \text { depot [poacher has laid the trap of clutch } \\
\text { wire] }\end{array}$ \\
\hline 54. & $\begin{array}{l}\text { February } \\
11,2010\end{array}$ & $\begin{array}{c}\text { Kirada-Lava Route (near Lava vil- } \\
\text { lage), Devprayag }\end{array}$ & - & Found dead, Trapped in fence \\
\hline
\end{tabular}




\begin{tabular}{|c|c|c|c|c|}
\hline 55. & $\begin{array}{l}\text { February } \\
12,2010\end{array}$ & $\begin{array}{l}\text { Jhajhra Forest (Majhaun), Dehradun } \\
\text { Forest Division ( } 200 \text { meters away } \\
\text { from Birodi village) }\end{array}$ & $\begin{array}{c}\text { Male, } 07 \\
\text { years }\end{array}$ & Found hanged in a tree in break-wire \\
\hline 56. & $\begin{array}{l}\text { February } \\
12,2010\end{array}$ & $\begin{array}{c}\text { Aasarodi Forest Range, Dehradun } \\
\text { Forest Division Near to Badowala } \\
\text { barrier (in private farm house) }\end{array}$ & $\begin{array}{l}\text { Female, } \\
06 \text { years }\end{array}$ & $\begin{array}{l}\text { Found dead, brutally captured in a trap (all four legs were } \\
\text { detached from body) }\end{array}$ \\
\hline 57. & $\begin{array}{l}\text { February } \\
15,2010\end{array}$ & $\begin{array}{l}\text { Rausaal village, Bhilangna Range, } \\
\text { Terai Forest Div. }\end{array}$ & $\begin{array}{l}\text { Female, } \\
02 \text { years }\end{array}$ & Found dead; died two days before \\
\hline 58. & $\begin{array}{l}\text { February } \\
15,2010\end{array}$ & $\begin{array}{l}\text { Chaka, Ludiyanaam Tok, Ghansora- } \\
\text { paka Forest }\end{array}$ & - & Found dead, unknown \\
\hline 59. & $\begin{array}{l}\text { February } \\
16,2010\end{array}$ & Langha Forest, Kalsi Forest Division & $\begin{array}{l}\text { Male, } 05 \\
\text { years }\end{array}$ & $\begin{array}{c}\text { Found dead; trap marks in neck portion, Nails missing from } \\
\text { three feet }\end{array}$ \\
\hline 60. & $\begin{array}{l}\text { February } \\
17,2010\end{array}$ & Beribara Forest, Rajaji National Park & $\begin{array}{l}\text { Male, } 01 \\
\text { year }\end{array}$ & Found dead \\
\hline 61. & $\begin{array}{l}\text { February } \\
19,2010\end{array}$ & $\begin{array}{l}\text { Back portion of IMA campus, De- } \\
\text { hradun attached }\end{array}$ & Female, - & $\begin{array}{c}\text { Captured in trap, rescued very late } \& \text { bring To with Tons river } \\
\text { Aasarodi but finally died [trap was placed for army training } \\
\text { purpose] }\end{array}$ \\
\hline 62. & $\begin{array}{l}\text { February } \\
23,2010\end{array}$ & Rikhrikhal Block, Pauri district & - & Found dead, Natural death? \\
\hline 63. & $\begin{array}{l}\text { February } \\
25,2010\end{array}$ & Ganja village, Bhimtal, Nainital & Female, - & $\begin{array}{l}\text { Found injured; rescued \& shifted to Nai Natal Zoo but finally } \\
\text { died }\end{array}$ \\
\hline 64. & $\begin{array}{l}\text { March 01, } \\
2010\end{array}$ & $\begin{array}{l}\text { Kotdwar Range, Lansdowne Forest } \\
\text { Division }\end{array}$ & - cub & Found dead; old carcass \\
\hline 65. & $\begin{array}{l}\text { March 08, } \\
2010\end{array}$ & $\begin{array}{c}\text { Near to Gaula river by-pass bridge, } \\
\text { Haldwani Forest Division }\end{array}$ & $\begin{array}{c}\text { Male, } 02 \\
\text { years }\end{array}$ & $\begin{array}{l}\text { Found dead; three claws were missing \& neck bone was } \\
\text { found fractured [looking like snow leopard] }\end{array}$ \\
\hline 66. & $\begin{array}{l}\text { March 12, } \\
2010\end{array}$ & $\begin{array}{l}\text { Belani, Near to Vidya Mandir, } \\
\text { Rudraprayag }\end{array}$ & $\begin{array}{c}\text { Female, } \\
01 \text { month }\end{array}$ & $\begin{array}{l}\text { Found dead; masticated spots found over To lower neck } \\
\text { portion }\end{array}$ \\
\hline 67. & $\begin{array}{l}\text { March 16, } \\
2010\end{array}$ & $\begin{array}{l}\text { Mandal Range, Jmeria Beat, Kalagarh } \\
\text { Tiger }\end{array}$ & $\begin{array}{l}\text { Female, } \\
02 \text { years }\end{array}$ & Found dead; Injured marks at neck, belly \& head \\
\hline 68. & $\begin{array}{l}\text { March 20, } \\
2010\end{array}$ & Saterakhal, Rudraprayag & $\begin{array}{l}\text { Female, } \\
02 \text { years }\end{array}$ & $\begin{array}{l}\text { Found dead; Poaching case [seriously Injured \& nails miss- } \\
\text { ing] }\end{array}$ \\
\hline 69. & $\begin{array}{l}\text { March 21, } \\
2010\end{array}$ & Khanpur, Haridwar Forest Division & - & Found dead \\
\hline 70. & $\begin{array}{l}\text { March 21, } \\
2010\end{array}$ & $\begin{array}{l}\text { Pantnagar Veterinary College, Pant- } \\
\text { nagar Shifted from Malsi Deer Park } \\
\text { for treatment }\end{array}$ & $\begin{array}{l}\text { Male, } 05 \\
\text { years }\end{array}$ & $\begin{array}{c}\text { Found trapped in a snare at Majri village, rescued \& shifted to } \\
\text { Malsi Deer Park, after that shifted to Pantnagar Veterinary } \\
\text { Col Lege for treatment; serious injury in neck Portion and } \\
\text { infection, finally died }\end{array}$ \\
\hline 71. & $\begin{array}{l}\text { March 21, } \\
\quad 2010\end{array}$ & Badowala, Dehradun Forest Division & - & $\begin{array}{c}\text { Found trapped in snare at Badowala vil lege, Badowala } \\
\text { village near Shimla bypass road Shimla bypass road; leopard } \\
\text { was captured during day hours [chest portion was seriously } \\
\text { injured] rescued \& shifted; finally died }\end{array}$ \\
\hline 72. & $\begin{array}{l}\text { March 27, } \\
2010\end{array}$ & $\begin{array}{l}\text { Kotawali forest, Chiriapur Range, } \\
\text { Haridwar Forest Division }\end{array}$ & $\begin{array}{l}\text { Female, } \\
02 \text { years }\end{array}$ & Road accident, Haridwar - Bijnor National Highway No. 74 \\
\hline 73. & $\begin{array}{l}\text { March 28, } \\
2010\end{array}$ & $\begin{array}{l}\text { Nirada/Mathana village, Pithoragarh } \\
\text { Forest Division }\end{array}$ & $\begin{array}{c}\text { Male, } 06 \\
\text { month }\end{array}$ & Found dead \\
\hline 74. & $\begin{array}{l}\text { March 28, } \\
2010\end{array}$ & $\begin{array}{l}\text { Nirada/Mathana village, Pithoragarh } \\
\text { Forest Division }\end{array}$ & $\begin{array}{l}\text { Female, } \\
08 \\
\text { months }\end{array}$ & Found dead \\
\hline 75. & $\begin{array}{l}\text { April 05, } \\
2010\end{array}$ & $\begin{array}{l}\text { Bohra village, Near Bhujaan, Civil } \\
\text { forest, Ranikhet Almora Forest Divi- } \\
\text { sion }\end{array}$ & Female, - & Found dead \\
\hline 76. & $\begin{array}{l}\text { April 10, } \\
2010\end{array}$ & Ramgarh Range, Rajaji National Park & - & Found dead, burnt in fire \\
\hline 77. & $\begin{array}{l}\text { April 10, } \\
2010\end{array}$ & $\begin{array}{c}\text { Sauni Forest, near to Pant gaon, } \\
\text { Ranikhet, Almora }\end{array}$ & Male, - & Found Dead, Unknown \\
\hline 78. & $\begin{array}{l}\text { April 11, } \\
2010\end{array}$ & Almora Forest Division & - & Brutally killed by villagers \\
\hline
\end{tabular}

Source: Self observations / news published in national level newspapers / official records. 


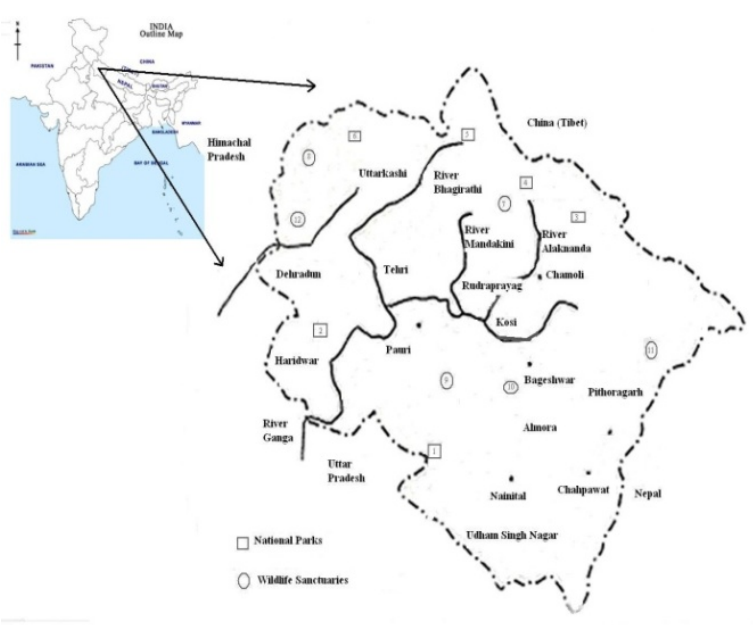

Figure 1. Protected habitats in Uttarakhand state (source: State Forest Department)

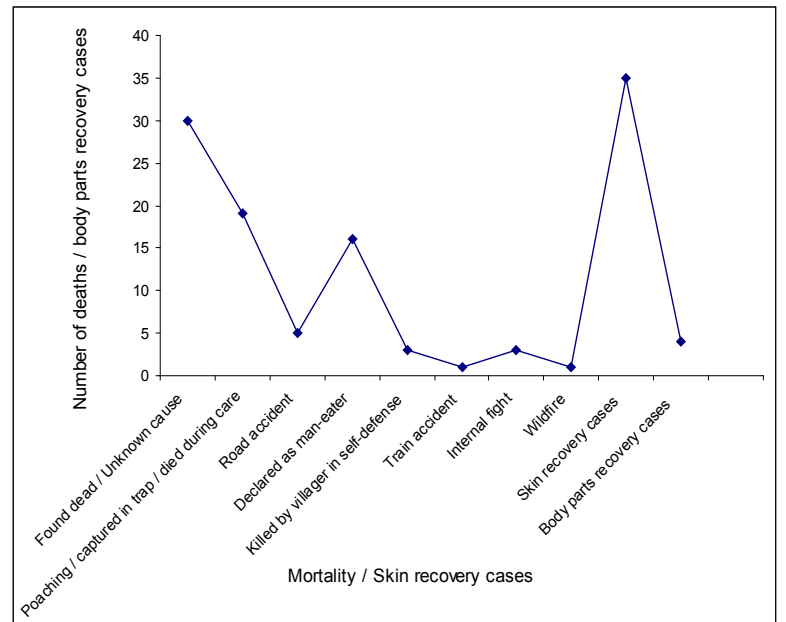

Figure 2. Mortality in protected leopard's population and skin recovery cases in Uttarakhand during January 2009 to April 11, 2010

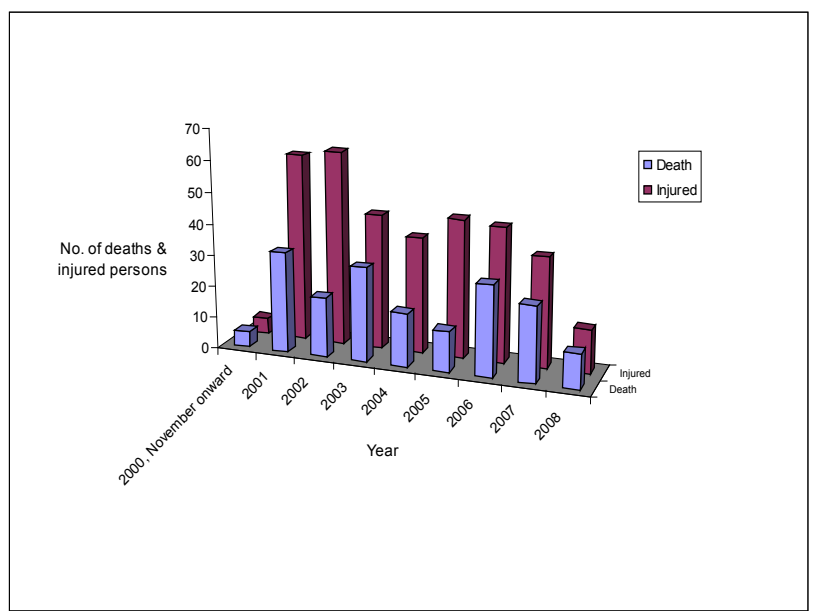

Figure 3. Number of human deaths and injured person in leopard's attack in Uttarakhand between November 2000 to December 2008 (Source: Data of Forest Department)

Leopard's mortality rate was significantly influenced by on-going poaching activities (trap knotting and poisoning for skin and body parts). During last two years, clutch-wire made snares were frequently found by the officials being used by the poachers, which also don't enable the animal to liberate out from trap. Notably, maximum number of deaths occurred during February 2009 \& 2010 (12) and March 2010 (11) followed by January 2010 (10). As most of the protected habitats are adjacent to villages and leopards used to move frequently in buffer zones and sometimes near to boundary of villages, therefore, instances of poaching cases are commonly observed from time to time (Figure 2).

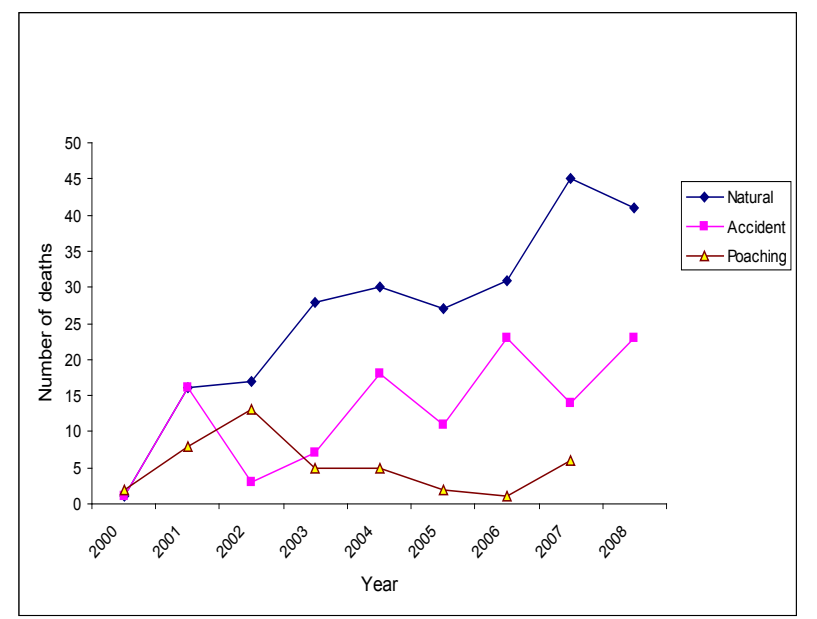

Figure 4. Leopard's death through various reasons at different parts of Uttarakhand during 2000 - 2008 (Source: Data of Forest Department)

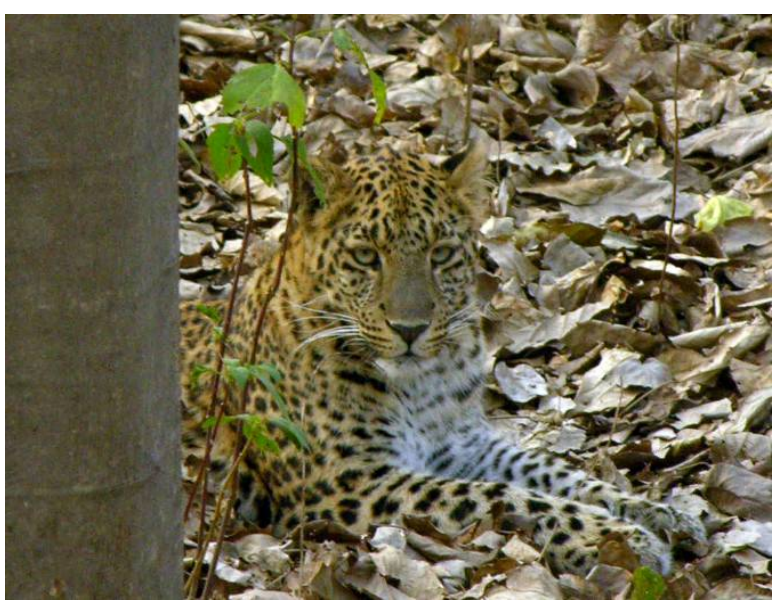

Figure 5A. Leopard in the Rajaji National Park

\section{Skin Recovery Cases}

Despite mortality cases, 35 leopard's skin were also recovered / seized by forest officials, civil police and special force at different locations of Uttarakhand during January 2009 to March 2010 (Table 2). Additionally, 04 other cases of recovering body parts (bones, nails \& teeth) were noted during said period. To control this, state forest department is conducting extensive patrolling and joint raid operations in sensitive areas besides, Task Forces are being constituted to check the illegal wildlife trade. Based on available figures, it can be said that poaching activities in Uttarakhand is effective and still ongoing (Figure $5 \mathrm{H} \& \mathrm{I}$ ). 
Table 2. Cases of leopard's skin recovery in Uttarakhand since January 2009

\begin{tabular}{|c|c|c|c|}
\hline S.No. & Date & Area & Case details \\
\hline 1. & $\begin{array}{l}\text { February } 08 \\
2009\end{array}$ & Haldwani & Bones of leopard recovered b Forest staff from Nepali person \\
\hline 2. & $\begin{array}{l}\text { February 14, } \\
2009\end{array}$ & Banbasa, Nepal Border & $\begin{array}{l}03 \text { skins were recovered by Seema Shastra Bal force from Nepal border; } 03 \text { people } \\
\text { ar rested }\end{array}$ \\
\hline 3. & $\begin{array}{l}\text { February 23, } \\
2009\end{array}$ & DakPathar are, Dehradun & $\begin{array}{c}\text { A skin was recovered by Special Task Force and Wildlife Protection Society of } \\
\text { India }\end{array}$ \\
\hline 4. & $\begin{array}{l}\text { March 18, } \\
2009\end{array}$ & Pokhri, Chamoli & 02 skins were recovered by Special Task Force and WPSI from Dehradun \\
\hline 5. & $\begin{array}{l}\text { March 23, } \\
2009\end{array}$ & Haridwar & Bones and Teeth of leopard were recovered by Haridwar Forest Division \\
\hline 6. & $\begin{array}{l}\text { April 25, } \\
2009 \\
\end{array}$ & Champawat & A skin was recovered by Special Operation Group at Champawat \\
\hline 7. & May 07, 2009 & Champawat & 02 skins were recovered by Champawat civil police \\
\hline 8. & June 20, 2009 & Badrinath area, Chamoli & A skin was recovered by Badrinath Forest staff \\
\hline 9. & June 29, 2009 & Haldwani & $\begin{array}{l}\text { Bones were recovered from Fatehpur, Haldwani/Ramnagar by Terai west forest } \\
\text { staff \& WPSI }\end{array}$ \\
\hline 10. & July 08, 2009 & Haridwar & 01 skin was recovered by Haridwar police \& forest division at Haridwar \\
\hline 11. & July 26, 2009 & Hailang, Birhi & 03 skins were recovered by Forest department in Chamoli district \\
\hline 12. & $\begin{array}{c}\text { September } \\
04,2009\end{array}$ & Nandprayag, Chamoli & 02 skins were recovered b Special Task Force \& WPSI; Poison/Poaching case \\
\hline 13. & $\begin{array}{c}\text { September } \\
12,2009\end{array}$ & Ghat, Chamoli & 01 skin was recovered by forest staff $\&$ WPSI \\
\hline 14. & $\begin{array}{c}\text { September } \\
14,2009 \\
\end{array}$ & Jakholi, Rudraprayag & 01 skin was recovered $\mathrm{b}$ forest staff \\
\hline 15. & $\begin{array}{l}\text { October 11, } \\
2009\end{array}$ & $\begin{array}{l}\text { Kalsi, Vikas nagar, De- } \\
\text { hradun }\end{array}$ & 02 skins were recovered b STF \& forest staff \\
\hline 16. & $\begin{array}{l}\text { November 11, } \\
2009\end{array}$ & Almora & 01 skin was recovered by Almora civil police \\
\hline 17. & $\begin{array}{l}\text { December } 21, \\
2009\end{array}$ & Haridwar & 05 skins were recovered by civil police of Haridwar \\
\hline 18. & $\begin{array}{l}\text { January } 07 \\
2010 \\
\end{array}$ & Rishikesh city area & 03 skins of leopard were recovered by Special Task Force of Rishikesh, Dehradun \\
\hline 19. & $\begin{array}{l}\text { January } 17, \\
2010\end{array}$ & Muni ki Reti (Tehri) & 01 skin of leopard was recovered by Special Operation Group, Rishikesh \\
\hline 20. & $\begin{array}{l}\text { February } 03, \\
2010\end{array}$ & $\begin{array}{l}\text { Lohaghat, Pithoragarh } \\
\text { village (Rawatgarah). }\end{array}$ & $\begin{array}{l}\text { Bones, tooth and nails of leopard were recovered by Seema Shastra Bal (SSB) near } \\
\text { Sail Nicotine Ganja was also recovered by poachers. }\end{array}$ \\
\hline 21. & $\begin{array}{c}\text { February } 13 \\
2010\end{array}$ & $\begin{array}{l}\text { Dineshpur More, } \\
\text { Rudrapur }\end{array}$ & $\begin{array}{l}\text { One leopard skin was recovered from poachers by SIDCUL Police \& SOG, } \\
\text { Rudrapur, U S Nagar [as per poachers they bring skin from Nepal and are going to } \\
\text { Haldwani for selling skin] }\end{array}$ \\
\hline
\end{tabular}

Source: self observations / news published in national newspapers / official records

Leopard's deaths during January 2009 - April 2010

\begin{tabular}{|c|c|}
\hline Found dead, unknown cause & 30 \\
Poaching / captured in trap (died during rescue / care) & 19 \\
Road accident & 05 \\
Declared as man-eater & 16 \\
Killed by villager in self-defense & 03 \\
Train accident & 01 \\
Internal fight & 03 \\
Wildfire & 01 \\
\hline
\end{tabular}

\section{Sex - ratio}

Out of 78 deaths, 24 were females whereas 15 were males and for other 39 the exact sex was not known to us. Looking into the data it comes out that the death ratio of male: female is almost equal and this will not affect the sex ratio of species but will bring this mammalian carnivore to threat.

\section{Status of Rescued Leopards}

Rescuing wild animal is a very sensitive and challenging task and needs very responsive treatment. Wild animals are very sensitive to human beings and proximity with them makes animal unconscious and furious. Besides, our efforts to rescue the leopards are not based on scientific protocol and that is one of the reason, which prolong deaths after rescue operations or during treatment. Out of 78 deaths, 12 were found trapped in snare and injured and shifted to some other safe areas but eventually all the leopards finally died. In addition, against to this, we have two examples in which leopards have been successfully rescued and translocated to 
some other places.

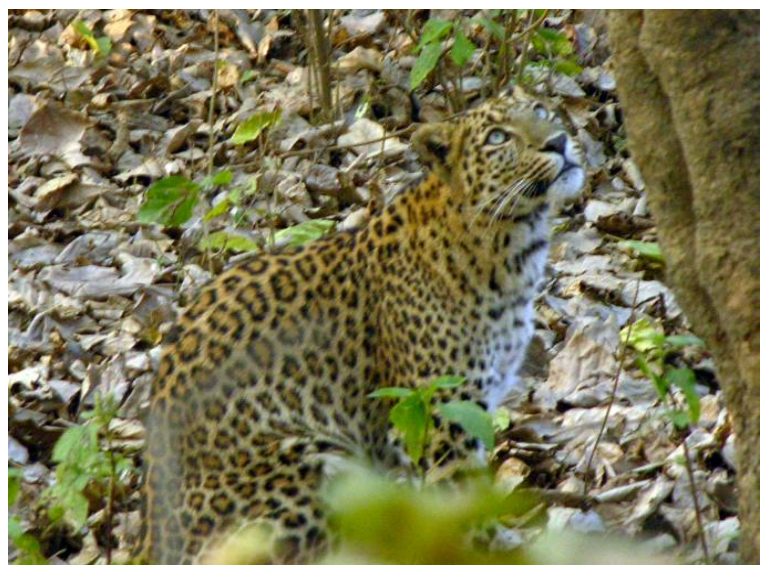

Figure 5B. Leopard about to climb the tree in Rajaji National Park

During January 2006, a male leopard was declared as man-eater in Badrinath forest division (Chamoli district) and after capturing him successfully from Budhadhang village, was released to Khara forest (Mitthawali area) of the Rajaji National Park. One such case had happened during 2008 in which a male leopard was translocated to Malsi Deer Park after being rescued from Dehradun-Mussoorie highway (Figure $5 \mathrm{~J}$ ). Man-eater leopards were captured from time to time through setting up of baited traps effectively but as far as on-spot rescue and treatment is concerned our efforts are unequipped to save wildlife.

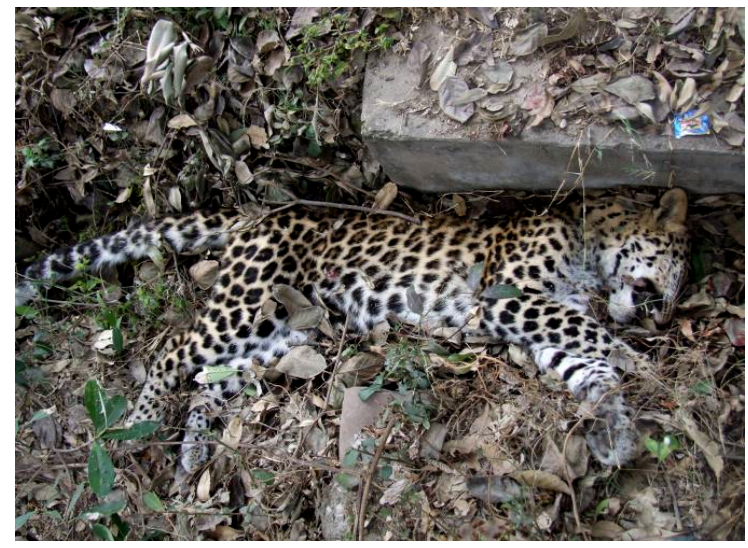

Figure 5C. Leopard's cub was found dead at Haridwar forest division

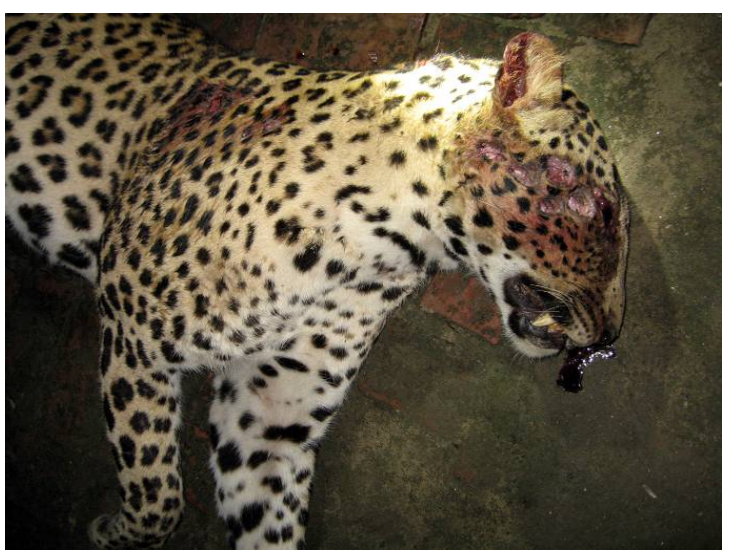

Figure 5D. Leopard brutally shot dead by poachers at Khanpur forest, Haridwar forest division during 2009

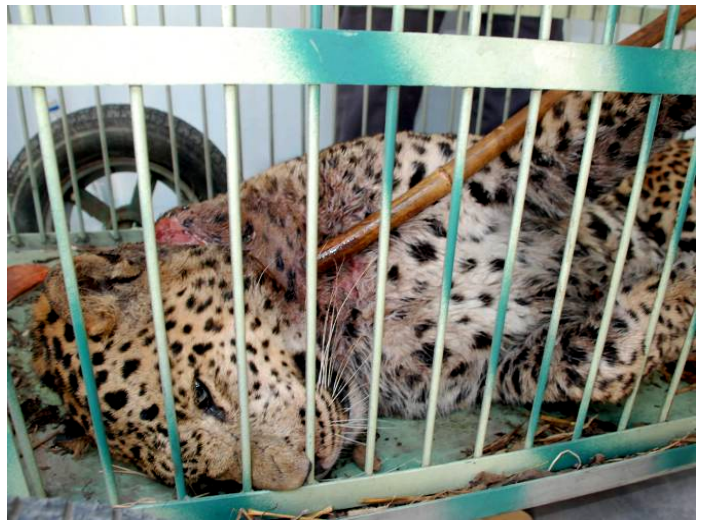

Figure 5E. Seriously injured male leopard at Malsi Deer Park after being rescued from Resam Mazri village, Dehradun during 2010; the neck portion was badly abraded due to caught in an illegal snare

\section{Rescue Center}

In an initiative to protect the state's endangered wildlife; state government initiated a project for care of rescued wild animals during 2010 especially for man-eater leopards. Under this project a rescue center has been established over an area of 25 hectares in Kotawali forest of the Haridwar forest division. Just after establishment four problem leopards were shifted to this centre from different locations of Garhwal Himalaya and are being providing treatment under supervision of experts. This initiative can be considered as a favourable step in wildlife conservation in north India. However, translocation of big cats is a controversial subject among various workers. In the event of a leopard problem, the most common management strategy followed throughout India is the setting up of baited traps, capture of an individual (not necessarily the problem causing individual) and its subsequent translocation into the nearest suitable natural habitat $[1,2]$. This is also recommended by the Indian Wildlife Protection Act[3] through an amendment made in 2002. However, this strategy is not recommended by scientists for managing problem animals because of the strong homing instincts exhibited by a wide range of carnivore families and the potential for movement of conflict with the individuals [1-4].

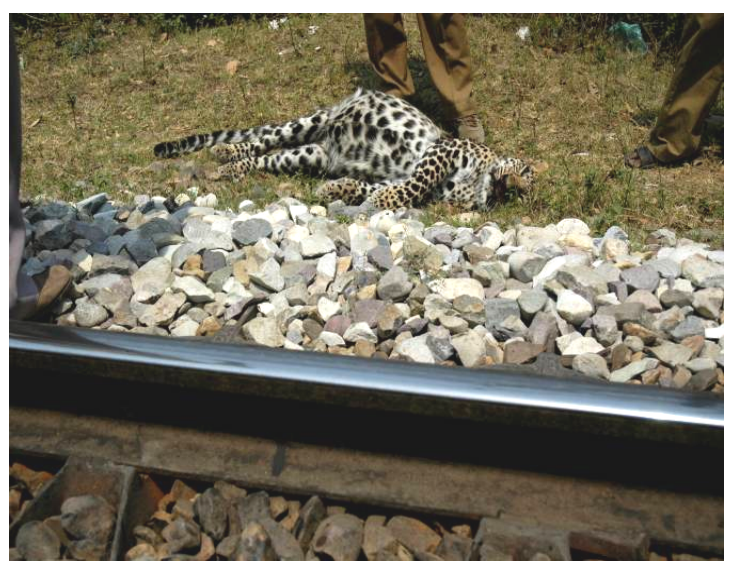

Figure 5F. Leopard died due to collision with train in Haridwar-Dehradun railway track at Motichur forest 


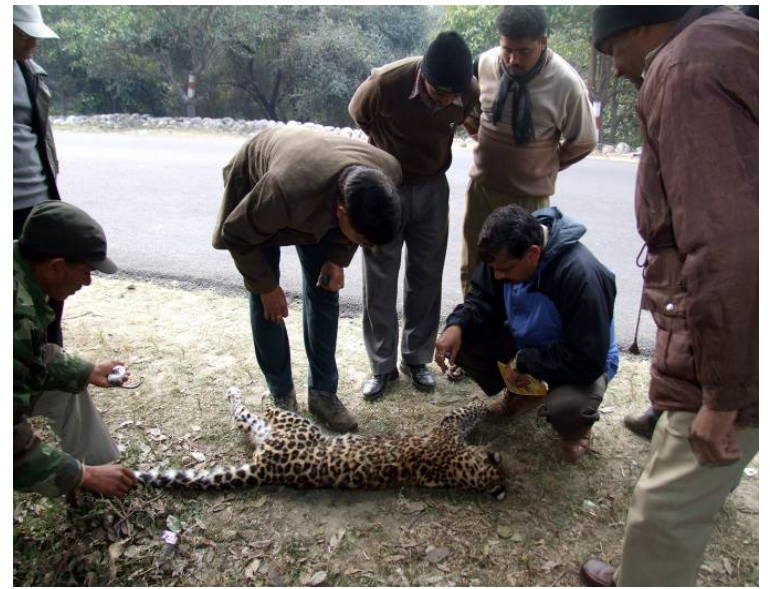

Figure 5G. Leopard died in a road accident at Haridwar-Bijnor National Highway No. 74, which is passing in between different forest zones

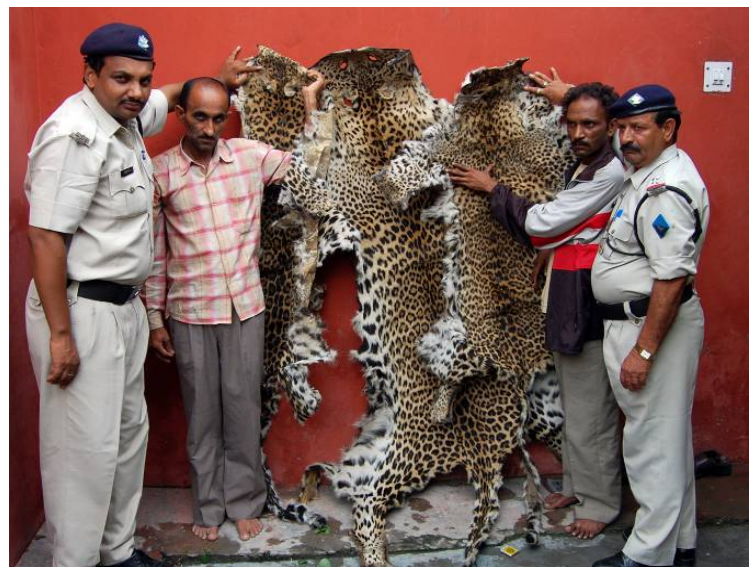

Figure 5H. Wildlife crime: Leopard's skins were recovered by civil police of Vikas Nagar, Dehradun during 2008

\section{Future of the Leopard's Population in Uttarakhand State}

Uttarakhand is rapidly losing its leopard wealth and therefore needs a very transparent attention and conservation actions. During November 2000 to December 2008, 180 people were killed by leopards and 343 were injured in leopard's attack (Figure 3). Notably, 394 leopards died due to various causes (236-natural, 116-accident and 42-poaching; Figure 4). On the other hand 50 leopards were declared as man-eater during November 2000 to December 2008 and most of them were shot down while some were shifted to other protected habitat (zoo, protected habitat \& zoological park). But maximum deaths accounted for shot due to fierce nature and demand of local people.

Maximum number of man-eaters were declared during 2009 (14) followed by 2006 and 2007 (11) and 2003 (09) whereas least number were declared during 2004 and 2005 (02). Historical literature revealed that 140 people succumbing to leopard attacks between 1988 and 2000 while 93 leopards were killed in the same period[5]. Since 2009, man-leopard conflict has been enhanced to two folds and today the situation in some places is very severe especially in rural areas.

Figures indicated that man-leopard conflict is increasing day-by-day mainly because of competition for resources and space. Most of the villages are situated in between the forest area and adjoining to protected habitats and wild animals (leopard, wild boar, bear, Himalayan yellow throated marten and several species of deer) used to move extensively near to these areas because of presence of water and palatable crops. Besides, traditionally villagers also used to enter nearby forests to collect fuel-wood and fodder year round and thus encounter rate is increasing. In this critical situation more effective action-oriented and scientific protocol based strategies are required for conservation of this endangered species, which should primarily involve the participation of local people at every level, like formulation of policies, government meetings, awareness programmes and research activities.

Uttarakhand has a long history of leopard problem especially man-eater, for which the world famous conservationist Jim Corbett is known. This problem is more common in hilly areas where leopard's movement is commonly observed adjoining to villages and currently its population is only safe in protected areas that don't consist of human habitation. Mammalian carnivores are vulnerable to local extinction in fragmented landscapes mainly due to their low densities, large ranges and inevitable conflict with humans[6,7].

Outside protected areas, accidental or intentional killing by people drives local extinction of large carnivores or reduces their numbers, and it is a matter of fact that many human-dominated landscapes will always be unsuitable for some large carnivores[8]. Animal-vehicle collisions have the potential to impact the viability of wildlife populations. Several studies have been carried out on mortality of wild animals[8-11].

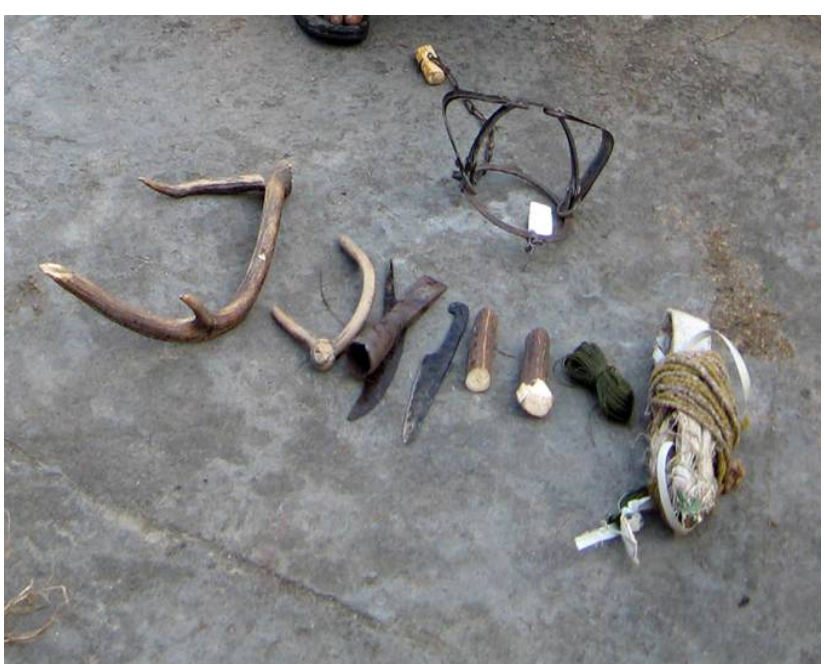

Figure 5I. Snare and other tools recovered by forest staff from poachers during a raid at Sonanadi Range, Kalagarh Tiger Reserve during 2009

Today, wildlife in several parts of India does not exist in isolation. Most wildlife populations live outside protected areas, often along side humans. Dogs, pigs and goats form an important part of leopards diet across many sites (Himachal 
Pradesh, Uttarakhand, Gujarat and Maharashtra) indicating that the feral animals and livestock are probably important prey of leopards living outside protected areas in India $[12,13]$.

In India, most of the national highways and railway tracks are also running across the potential protected wildlife habitats, which impede the frequent movement of animals within their home range. In this situation there is further need to conduct more scientific studies on the unnatural deaths of wildlife to address the requirements of both humans and wild fauna.

\section{Recommendations}

1. Proper census should be carried out at potential sites especially in buffer zones, where leopard's movement is very common and at risk.

2. At some places locals use to place snare / trap during night near to boundaries of the protected areas, therefore night patrolling of adjoining villages will be beneficial to know the status of poaching activities.

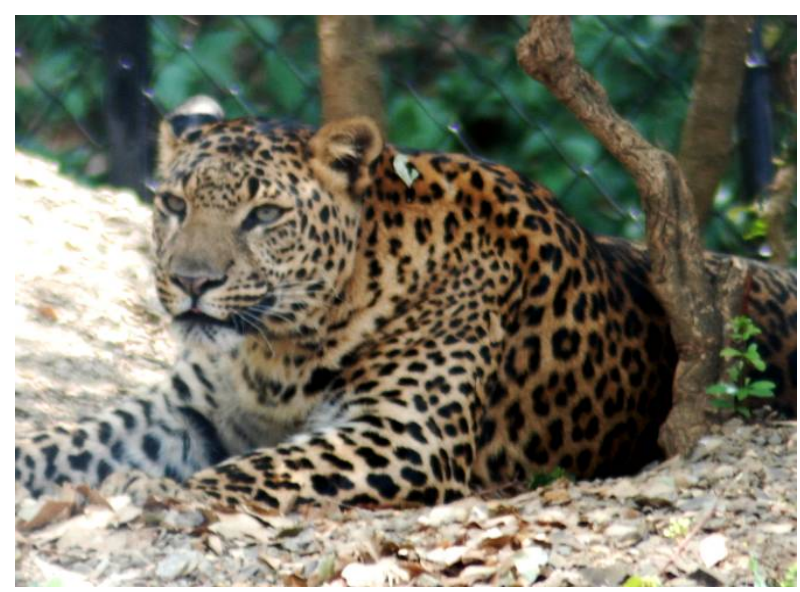

Figure 5J. Towards conservation: A translocated leopard-Raja, rescued from Dehradun-Mussoorie highway and released into Malsi Deer Park, Dehradun

3. As most of the villagers are living adjacent to the protected habitats traditionally and their indigenous knowledge about the forest resources and wildlife conservation is important to be addressed, therefore if we incorporate their opinions in making conservation policy that will be immensely helpful in conservation of wildlife wealth.

4. Community participation should be ensured besides; involvement of local people, government organisation, NGOs and subject experts is highly required.

5 Affected regions should be monitored sharply and movement of other wild animals (herbivores) should be ensured. It will be helpful in knowing the specific status of poaching of wildlife.

6 Death reason should be explored properly so that we should know the accurate death reason and be cautious in future about that.

7 Radio-telemetric studies are required on leopards, which were released / translocated to protected habitat. This will be helpful in knowing the adaptation status of species and on the basis of which some recommendations could be given.

\section{ACKNOWLEDGEMENTS}

We are thankful to I am thankful to Mr. Hem Pande, Joint Secretary, Dr. Sujata Arora, Director, Ministry of Environment and Forests, Govt. of India and to Dr. Rambir Singh, Director, Ministry of Science and Technology, Govt. of India for their encouragement and valuable suggestions. $\mathrm{Mr}$. C.M. Dobhal, Director and Mr. O.P. Bhatt, Chairman of the Doon Institute of Engineering and Technology, Uttarakhand and to various forest officers and media persons for providing suggestions and relevant information.

\section{REFERENCES}

[1] V.R. Athreya, S.S. Thakur, S. Chaudhuri, and A.V. Belsare, 'A study of the man-leopard conflict in the Junnar forest division, Pune district, Maharashtra'. Technical Report, 2004. [Online]. http://www.ncra.tifr.res.in/ rathreya/JunnarLeopards

[2] V.R. Athreya, and A.V. Balsare, Human-leopard conflict management guidelines. Technical Report, Kaati Trust, Pune, India (2007)

[3] Anon, The Wildlife (Protection) Act, 1972. As amended by The Wildlife (Protection) Amendment Act, 1972. Professional Book Publishers, New Delhi, India (2002)

[4] Linnell, J.D.C., Aanes, R., Swenson, J.E., Odden, J., and Smith, M.E., 1997, Translocation of carnivores as a method for managing problem animals: a review., Biodiversity and Conservation, $6,1245-1257$

[5] D.S. Chauhan, and S.P. Goyal, A study on distribution, relative abundance and food habits of leopard (Panthera pardus) in Garhwal Himalayas. Technical Report, Wildlife Institute of India, Dehradun, India (2000)

[6] Noss, R.F., Quigley, H.B., Hornocker, M.G., Merill, T., and Paquet, P.C., 1996, Conservation biology and carnivore conservation in the Rocky Mountains., Conservation Biology, 10, 949-963

[7] Woodroffe, R., and Ginsberg, J.R., 1998, Edge effects and extinction of populations inside protected areas., Science, 280, 2126-2128

[8] Balme, G., and Hunter, L., 2004, Mortality in a protected leopard population, Phinda Private Game Reserve, South Africa: A population in decline?, Ecological Journal, 6, 1-6

[9] Singh, A.P., and Sharma, R.C., 2001, Conflicts between linear developments and Asian elephants in sub-Himalayan zone of Uttaranchal. In: Ecology and transportation (Eds. C.L. Irwin, P. Garrett, and K.P. McDermott), Centre for Transportation and the Environment, North Carolina State University, Raleigh, NC, 423-432

[10] Joshi, R., and Joshi, B.D., 2000, On the recurrence of rail accident death of an elephant (Elephas maximus) in Haridwar 
range of the Rajaji National Park., Him. J. Env. Zool., 14(2), $123-128$

[11] A.K. Singh, A. Kumar, A. Mookerjee, and V. Menon, Jumbo Express - A scientific approach to understanding and mitigating elephant mortality due to train accidents in Rajaji National Park. Technical Report, Wildlife Trust of India, Delhi, India (2001)
[12] Mukherjee, S., and Mishra, C., 2001, Predation by leopard Panthera pardus in Majhatal Harsang Wildlife Sanctuary, W. Himalayas., Journal Bombay Natural History Society, 98, 267-268

[13] A. Edgaonkar, and R. Chellam, A preliminary study on the ecology of the leopard Panthera pardus fusca in Sanjay Gandhi National Park, Maharashtra. Technical Report, Wildlife Institute of India, Dehradun, India (1998) 